E R R A T A

OFF-DIAGONAL LONG-RANGE ORDER IN HEISENBERG FERROMAGNET. S. P. Heims [Phys. Rev. Letters 13, 50 (1964)].

The statements in the Letter are ambiguous, because the nature of the zero-field limit was not specified. The rigorous and unambiguous statements for the Heisenberg ferromagnet are these: ODLRO exists for $T<T_{c}$ in the limit $H \rightarrow 0, N \rightarrow \infty, N \mu H / k T \rightarrow$ constant or zero. In this limit, which is the analog to the superfluid state of $\mathrm{He}^{4}$, the magnetization is zero. In the limit $H \rightarrow 0, N \rightarrow \infty$, and $N \mu H / k T \rightarrow \infty$ no ODLRO exists, but magnetization is finite for $T<T_{c}$. The order parameter

$$
\lim _{|i-j| \rightarrow \infty}\left\langle\sigma_{z}^{i}{ }_{\sigma}^{j}{ }_{z}^{j}\right\rangle
$$

is always finite for $T<T_{C}$ as $H \rightarrow 0$ and $N \rightarrow \infty$.

SU(6) CLEBSCH-GORDAN COEFFICIENTS FOR THE PRODUCT $35 \times 56$. J. C. Carter, J. J. Coyne, and S. Meshkov. [Phys. Rev. Letters 14, $523(1965)]$.

Table I has incorrect entries which should be replaced by the values listed in the following table:

\begin{tabular}{|c|c|c|c|c|c|}
\hline & & $\left(\underline{8}^{3} \times 10^{4}\right)$ & $\left(\underline{8}^{1} \times \underline{10}^{4}\right)$ & $\left(\underline{8}^{1} \times \underline{8}^{2}\right)_{s}$ & $N$ \\
\hline & ${\frac{35^{4}}{27^{2}}}^{2}$ & $\sqrt{3}$ & $\sqrt{5}$ & $-\sqrt{3}$ & \\
\hline$\underline{1134}$ & $\begin{array}{l}\frac{35}{8}^{4} C^{4} \\
\frac{27}{A} A^{2} \\
\underline{27}_{B}{ }^{2}\end{array}$ & $-\sqrt{5}$ & $\begin{array}{r}\sqrt{3} \\
-9\end{array}$ & $\frac{-1}{\sqrt{3}}$ & $\sqrt{12}$ \\
\hline
\end{tabular}

Only the phase changes in the $\left(8^{1} \times \underline{8}^{2}\right)_{s}$ entries for the $27^{2}$ representations affect the results quoted in the paper. These introduce an obvious phase change in Eqs. (8) and (9), and reverse the phases of the $K^{+} p$ amplitudes of Eq. (11). Equation (12) should be replaced by the relation

$$
\begin{aligned}
\left(\pi^{+} p \mid K^{+} \Sigma^{+}\right) & =-\sqrt{3}\left(\pi^{+} p \mid K^{*+} \Sigma^{+}\right) \\
& =\left(6^{1 / 2} / 4\right)\left(\pi^{+} p \mid K^{*+} Y^{*+}\right) .
\end{aligned}
$$

The altered and new $\pi^{+} p$ amplitudes should read

$$
\begin{aligned}
\left(\pi^{+} p \mid \rho^{+} p\right)=(-\sqrt{3} / 48) \underline{70} & +(\sqrt{3} / 6) \underline{700} \\
+ & (-7 \sqrt{3} / 48) \underline{1134},
\end{aligned}
$$

$$
\begin{gathered}
\left(\pi^{+} p \mid \rho^{0} N^{*++}\right)=\left(\frac{1}{8}\right) \underline{70}+\left(-\frac{1}{4}\right) \underline{700}+\left(\frac{1}{8}\right) \underline{1134}, \\
\left(\pi^{+} p \mid K^{*+} \Sigma^{+}\right)=(\sqrt{3} / 48) \underline{70}+(-\sqrt{3} / 48) \underline{134}, \\
\left(\pi^{+} p \mid K^{*+} Y^{*+}\right)=\left(-6^{1 / 2} / 24\right) \underline{70}+\left(6^{1 / 2} / 24\right) \underline{1134} .
\end{gathered}
$$

MESON AND BARYON MASSES IN SU(6) SYMMETRY. H. Harari and H. J. Lipkin [Phys. Rev. Letters 14, 570 (1965)].

Equation (1) should be replaced by

$$
4 \frac{(\rho-\pi)-\left(K^{*}-K\right)}{\rho-2 \pi-3 \eta+2(\omega+\varphi)-8\left(K^{*}-K\right)}=\frac{\Sigma-\Lambda}{\Xi^{*}-\Xi} .
$$

The value of the left-hand side should be replaced by $0.04 \pm 0.04$.

We thank Dr. W. Alles for pointing out this error.

\section{ALGEBRA OF CURRENT COMPONENTS AND THEIR MOMENTS: AN INTERPRETATION OF} SU(6). Benjamin W. Lee [Phys. Rev. Letters 14, 673 (1965)].

Reference 17 is misleading and we wish to clarify this. There are three solutions to Eq. (7): (1) the trivial solution $G_{\xi}=A_{\xi}=\left\langle r_{p}{ }^{2}\right\rangle=\left\langle r_{n}{ }^{2}\right\rangle=0$; (2) the solution exhibited; (3) the solution characterized by $D / F=-\frac{3}{2}$ and $\left\langle r_{n}{ }^{2}\right\rangle \neq 0$.

When one considers the commutation relation between the $A_{i}(\lambda)$ and $\mathfrak{T}_{j}(\mu)$, and assumes that the matrix elements of $A_{i}(\lambda)$ are given correctly by the considerations on page 677 , one finds that the only consistent solution is of the first kind. This is in agreement with the observation of Gell-Mann (see reference 11) that, if the baryon states form a 56-dimensional representation of the current algebra with $L=0$, the matrix element of $\mathfrak{M}_{j}(\mu)$ between two baryon states must be zero. The assumption that the intermediatestate sum in Eq. (3) is saturated by the 56 -plet of baryons implies that $L=0$ for these states. Gell-Mann shows further that if we consider the perturbation due to the kinetic-energy term $\mathfrak{H}^{\prime \prime}$, then the effective magnetic-moment operator transforms according to the adjoint representation with $L=0$. The solution exhibited is the unique solution when this information is adjoined to $\mathrm{Eq} .(7)$. 